\title{
I. Die Fundamente: Fürsorge in der Weimarer Republik und im Nationalsozialismus
}

Bis zur Verabschiedung des BSHG basierte das Fürsorgesystem in den Westzonen bzw. in der Bundesrepublik auf Fundamenten, die bereits in der Weimarer Republik gelegt worden waren. Die „Verordnung über die Fürsorgepflicht“ (RFV) vom 13. Februar 1924, die „Reichsgrundsätze über Voraussetzung, Art und Maß der öffentlichen Fürsorge“ (RGr.) vom 4. Dezember 1924 sowie das „Reichsgesetz für Jugendwohlfahrt" (RJWG) vom 9. Juli $1922^{1}$ hatten die Grundlagen für eine moderne Wohlfahrtspflege gelegt. Sie spiegeln die Entwicklung von der traditionellen Armenfürsorge hin zu einem ausdifferenzierten System öffentlicher Wohlfahrtspflege wider, die im Kaiserreich begonnen hatte, durch den Ersten Weltkrieg forciert und durch die Weltwirtschaftskrise vorläufig beendet wurde. ${ }^{2}$ Die RFV, die das formelle Fürsorgerecht regelte, schrieb eine öffentliche Fürsorgepflicht fest, faßte die bisherige zersplitterte Spezialgesetzgebung weitgehend zusammen, gab der öffentlichen Fürsorge eine einheitliche organisatorische und finanzielle Basis und ermöglichte eine stärkere Normierung der Unterstützungsgrundsätze. ${ }^{3}$ Beibehalten wurde die für die Stellung der Fürsorge im gesamten Sicherungssystem charakteristische prinzipielle Verpflichtung des Hilfsbedürftigen, die erhaltene Unterstützung später zurückzuerstatten.

$\mathrm{Zu}$ den Aufgaben der kommunalen Fürsorge gehörte neben der traditionellen Armenfürsorge nun auch die Fürsorge für Kriegsopfer, für Sozial- und Kleinrentner, für Schwerbeschädigte und Schwererwerbsbeschränkte sowie für Schwangere und Wöchnerinnen. Statt der oft leistungsschwachen (Orts-)Armenverbände hatten die Länder jetzt leistungsfähige Bezirks-, außerdem Landesfürsorgeverbände (BFV und LFV) als Träger zu bestimmen; zu Bezirksfürsorgeverbänden machten die Länder von da an meist nur noch Stadt- und Landkreise, während die überörtliche Trägerschaft in Preußen von den Provinzial- oder entsprechenden höheren Kommunalverbänden, sonst meist von den Ländern übernommen wurde. ${ }^{4}$ Die Zuständigkeit wurde durch die RFV erheblich vereinfacht: Nicht mehr der „Unterstützungswohnsitz“, der oft aufwendige Ermittlungen der jeweiligen Äm-

\footnotetext{
RFV: RGBl. 1924 I, S. 100; RGr.: RGBl. 1924 I, S. 765; RJWG: RGBl. 1922 I, S. 633, 647.

2 Zur Geschichte der Wohlfahrtspflege in der Weimarer Republik und ihrer Pervertierung während des Nationalsozialismus vgl. grundlegend Sachße/Tennstedt, Geschichte, Bd.2 und Bd. 3; für Weimar außerdem (mit erstaunlich geringer Bezugnahme zu Sachße/Tennstedt) Hong, Welfare, sowie Crew, Germans. Einen Forschungsüberblick für den Untersuchungszeitraum 1890-1945 geben Rudloff, Souterrain, und Andreas Wollasch, Tendenzen; weitere Literaturhinweise auch bei Willing, Vorgeschichte, S. $589 \mathrm{ff}$.

3 Vgl. ausführlich Sachße/Tennstedt, Geschichte, Bd.2, S. 142ff., $173 \mathrm{ff}$.

4 In Hessen, Baden, Sachsen, Thüringen und den Stadtstaaten fungierten die Länder als Landesfürsorgeverbände, in Bayern hingegen für die meisten Aufgaben die Kreise; Württemberg und Anhalt bildeten gesonderte Landesfürsorgeverbände aus den Bezirksfürsorgeverbänden; vgl. Memelsdorff, Träger, S. 684ff.; NDV 30 (1950), S. $266 f$.
} 
ter erfordert hatte, sondern der „gewöhnliche Aufenthalt" des Hilfsbedürftigen entschied jetzt, welcher Fürsorgeverband am Ende die Unterstützung zu zahlen hatte.

Ein Kernstück der Reformgesetzgebung betraf die Finanzierung der Fürsorge: Nachdem bisher das Reich den Hauptteil der kriegs- und inflationsbedingten Sonderfürsorgen finanziert hatte, wälzte es diese Lasten jetzt auf Länder und Kommunen ab, ohne allerdings längerfristig den Kommunen entsprechende Einnahmequellen zur Verfügung zu stellen. ${ }^{5}$ Für die weitere Entwicklung sehr bedeutsam war schließlich die Aufwertung der freien Wohlfahrtspflege: $\ 5$ RFV sah nicht nur die Möglichkeit vor, öffentliche Fürsorgeaufgaben an freie Träger zu delegieren, sondern enthielt auch die bekannte Bestandsgarantie, wonach keine Einrichtungen der öffentlichen Fürsorge geschaffen werden sollten, soweit geeignete Einrichtungen der freien Wohlfahrtspflege ausreichend vorhanden waren.

In den RGr. wurde die konkrete materielle Ausgestaltung der Fürsorge erstmals reichseinheitlich näher geregelt und damit gravierend in eine gemeindliche Prärogative eingegriffen. Die Hilfe hatte sich grundsätzlich auch weiterhin an der individuellen Notlage des Hilfsbedürftigen zu orientieren und sollte vor allem Hilfe zur Selbsthilfe im Sinne wirtschaftlicher Selbständigkeit sein. Entsprechend einem gewandelten Armutsverständnis war als Lebensbedarf nun nicht mehr nur das unbedingt Lebensnotwendige zu gewähren, sondern darüber hinaus auch das, was zur Erhaltung und (Wieder-)Herstellung von Gesundheit und Arbeitsfähigkeit nötig war. ${ }^{6}$ Dafür konnte die Hilfe auch vorbeugend gewährt werden. Daneben behielt die Weimarer Fürsorgegesetzgebung allerdings einen privilegierten Bereich in Form der sogenannten gehobenen Fürsorge für die kriegs- und inflationsbedingten Notlagen der Kriegsbeschädigten, Sozialrentner etc. mit günstigeren finanziellen Regelungen sowie besonderen Integrationshilfen für die Kriegsopfer („,soziale Fürsorge“) bei.7 Indirekt wurde dadurch eine weitere „,soziale Ausgestaltung' der Fürsorge"8 gefördert, indem in diesen Bereichen verwirklichte Unterstützungsgrundsätze und Methoden vor allem nach dem Zweiten Weltkrieg auf die gesamte Fürsorge ausstrahlten.

Der mit RFV und RGr. begonnene Trend hin zu einer stärkeren Vereinheitlichung der Fürsorgeleistungen wurde gegen den Widerstand der Kommunen 1925/26 fortgeführt, indem die Fürsorgeverbände dazu verpflichtet wurden, die

5 Vgl. Sachße/Tennstedt, Geschichte, Bd.2, S. 175ff. Vor allem in den Haushalten der größeren Städte entwickelte sich infolge neuer wohlfahrtspflegerischer Standards das Wohlfahrtswesen zum größten Ausgabenposten und betrug kurz vor der Weltwirtschaftskrise durchschnittlich ein Drittel des reinen Finanzbedarfs; vgl. Leibfried/Hansen/Heisig, Politik, S. $153 \mathrm{ff}$.

6 Vgl. ausführlich die amtliche Erläuterung zu $\$ 6$ RGr., abgedruckt in: Muthesius, Grundlagen, S. 84.

7 Gleichsam ein Gegenstück zur „gehobenen Fürsorge“ war die in $\ 13$ RGr. vorgesehene Möglichkeit, bei „Arbeitsscheu oder offenbar unwirtschaftlichem Verhalten [...] Art und Maß der Fürsorge auf das zur Fristung des Lebens Unerläßliche zu beschränken“; auf diese Weise wurden „die ,Asozialen“ als lumpenproletarische Unterstockgruppe innerhalb der Armenfürsorge neu eingeführt, und die Regelfürsorge war dadurch zugleich aufgewertet"; Sachße/Tennstedt, Geschichte, Bd. 2, S. 174.

8 Ebenda, S. 149. 
Höhe der Unterstützung auf der Basis von Richtsätzen zu bemessen. ${ }^{9}$ Wenn auch weder Berechnungsgrundlagen noch die tatsächliche Höhe der Richtsätze vorgeschrieben und vereinheitlicht waren, lief diese Politik „im Grunde auf die reichseinheitliche Garantie eines gewissen Mindesteinkommens für Hilfsbedürftige “10 hinaus.

Wie RFV und RGr. sollte auch das im Juni 1922 verabschiedete RJWG unterschiedliche organisatorisch-administrative und materielle Entwicklungen reichsweit vereinheitlichen. ${ }^{11}$ In seiner ursprünglichen Fassung sah das Gesetz eine erhebliche Ausweitung der kommunalen Aufgaben vor und schuf Grundstrukturen, die bis heute das Recht der Jugendhilfe bestimmen. Das RJWG statuierte einen Rechtsanspruch des Kindes auf Erziehung und erklärte neben der Jugendfürsorge erstmals auch die Jugendpflege zur Aufgabe der kommunalen Jugendhilfe, die durch selbständige Jugendämter in enger Fühlungnahme mit den freien Trägern durchzuführen sei. ${ }^{12}$ Doch noch vor seinem für 1924 geplanten Inkrafttreten wurde das Innovationspotential des RJWG empfindlich reduziert. Die Kommunen fürchteten vor allem die finanziellen Mehrbelastungen und verhinderten mit massiven Protesten, daß sie zur Errichtung eigener Jugendämter und zur Durchführung jugendpflegerischer Aufgaben verpflichtet wurden; die wirtschaftliche Minderjährigenfürsorge wurde durch die RFV der allgemeinen Fürsorge zugeschlagen. ${ }^{13}$ Nichtsdestoweniger führte auch das derartig reduzierte RJWG, das am 1. April 1924 in Kraft trat, zu einer erheblichen Ausdehnung öffentlicher Erziehungskompetenzen, zu einem weiteren freiwilligen Ausbau der Jugendämter und $\mathrm{zu}$ verstärkten Aktivitäten der Kommunen in der Jugendpflege. ${ }^{14}$

Für die Entwicklung der Wohlfahrtspflege in der Weimarer Republik bis zur Weltwirtschaftskrise insgesamt machen Christoph Sachße und Florian Tennstedt neben dem Trend zur Zentralisierung, Bürokratisierung und Professionalisierung folgende zentrale Spannungsmomente aus ${ }^{15}$, deren Folgen zum Teil bis in die Fürsorge der jungen Bundesrepublik hineinwirkten: 1. Die Öffnung der Wohlfahrts-

9 Vgl. „Verordnung zur Änderung der Reichsgrundsätze über Voraussetzung, Art und Maß der öffentlichen Fürsorge“ vom 7.9.1925, RGBl. 1925 I, S.332, sowie „Gesetz über Abänderung der Reichsverordnung über die Fürsorgepflicht“"vom 8. 6. 1926, RGBl. 1926 I, S. 255. Zur Entwicklung der Richtsätze als zentraler Größe in der Fürsorgepolitik und über diese hinaus (Richtsätze als Maßstab für Armut wie auch für die kommunalen Fürsorgeausgaben) vgl. Leibfried, Existenzminimum; ferner Leibfried/Hansen/Heisig, Geteilte Erde; Sachße/Tennstedt, Geschichte, Bd.2, S. $179 \mathrm{ff}$.

10 Sachße/Tennstedt, Geschichte, Bd.2, S. 149.

$11 \mathrm{Zu}$ Entstehung und Entwicklung des RJWG vgl. ausführlich ebenda, S. 99ff., sowie Hasenclever, Jugendhilfe, S. 48ff., 73ff.; Andreas Wollasch, Fürsorgeverein, S. 122ff. Zur Geschichte der deutschen Jugendfürsorge bis 1932 und speziell zur problematischen Praxis bei der Zwangserziehung vgl. Peukert, Grenzen, sowie die bei Rudloff, Souterrain, S. 499ff., besprochenen jüngeren Arbeiten.

12 Außerdem regelte das Gesetz das elterliche Erziehungsrecht, den Schutz der Pflegekinder, vormundschaftliche Aufgaben des Jugendamts sowie Schutzaufsicht und Fürsorgeerziehung.

13 Vgl. Verordnung über das Inkrafttreten des Reichsgesetzes für Jugendwohlfahrt, RGBl. 1924 I, S. 110.

14 Vgl. Sachße/Tennstedt, Geschichte, Bd. 2, S. 105f., $109 \mathrm{ff}$.

15 Vgl. ebenda, S. $213 \mathrm{ff}$. 
pflege hin zum Mittelstand im Zuge von Weltkrieg und Inflation, die eine erhebliche Heterogenität der „Armutsklientel“, damit auch der Fürsorgepraxis bedingte und den Widerspruch zwischen Standardisierung und Individualisierung der Fürsorgeleistungen verschärfte. 2. Den grundlegenden Konflikt zwischen Reich und Kommunen, in dem sich wohlfahrts- und finanzpolitische Motive eng verschränkten. Während das Reich immer mehr Steuerungskompetenzen für sich beanspruchte und fürsorgerische Standards reichsweit vorgab, sahen die Gemeinden und Gemeindeverbände ihre Autonomie auf einem ihrer ureigenen Betätigungsfelder schwinden. Dazu parallel verlief der Prozeß eines finanziellen Autonomieverlusts der Gemeinden im Zuge der Erzbergerschen Finanzreform, die sie zu „Kostgängern“ des Reiches machte, das ihnen mit der neuen Fürsorgegesetzgebung gleichzeitig neue Lasten auferlegte. Aus kommunaler Sicht sind seitdem Fürsorgeund Finanzpolitik die beiden Kehrseiten ein und derselben Medaille geblieben. ${ }^{16}$ 3. Die Bildung der „dualen“ Struktur der Wohlfahrtspflege, in der sich die freien Verbände parallel zu „Verreichlichung“ und Bürokratisierung des öffentlichen Fürsorgesektors zu professionellen Großverbänden entwickelten und mit der öffentlichen Wohlfahrtspflege einen „wohlfahrtsindustriellen Komplex“ bildeten. 4. Ein weiterhin paternalistisches Selbstverständnis der Wohlfahrtspflege, das den Prinzipien eines demokratisch legitimierten Wohlfahrtsstaates im Grunde widersprach. 5. Die kontinuierliche Orientierung der Wohlfahrtspflege am Arbeitsmarkt; zentrales Ziel der Fürsorgearbeit blieb in all ihren Bereichen die (Wieder-) Eingliederung in das Erwerbsleben, was in Zeiten hoher, zunehmend auch struktureller Arbeitslosigkeit das Wohlfahrtssystem in die Krise führen mußte.

Tatsächlich gingen dann zahlreiche der zukunftsweisenden Ansätze in den Massennotlagen der Weltwirtschaftskrise seit Ende 1929 unter: Millionen Wohlfahrtserwerbslose, für deren Unterstützung immer mehr die Kommunen aufzukommen hatten, konterkarierten die Bemühungen um eine individualisierende und rehabilitative Fürsorge. An ihre Stelle trat eine zunehmend repressive, standardisierte Minimalsicherung, die Städte und Gemeinden trotz rigider Sparmaßnahmen an den Rand des finanziellen Ruins brachte, so daß die Abhängigkeit der Kommunen vom Reich schon vor 1933 weit fortgeschritten war. ${ }^{17}$

Während der Zeit des Nationalsozialismus blieben die rechtlichen Grundlagen der Fürsorge im wesentlichen unangetastet, de facto erlebte die Wohlfahrtspflege jedoch einen tiefen Umbruch im Zeichen der Förderung der „Wertvollen“ einerseits, der Aussonderung der „Minderwertigen“ bis hin zu deren Ermordung andererseits. Bezugspunkt einer „erbbiologischen“ und „bevölkerungspolitischen“ Grundsätzen verpflichteten nationalsozialistischen Fürsorge war nicht mehr die Hilfe für das schwache Individuum, sondern die Pflege des „gesunden Volkskörpers“. ${ }^{18}$ Das bedingte grundsätzlich eine Aufwertung der Wohlfahrtspflege zur

16 Sinnfällig wird diese Verknüpfung schon in der Tatsache, daß die RFV zum Vollzug der Dritten Steuernotverordnung verkündet wurde. $\mathrm{Zu}$ diesem Komplex vgl. insgesamt auch Münch, Sozialpolitik, S. 66ff.

17 Vgl. Sachße/Tennstedt, Geschichte, Bd. 3, S. 64ff., 84ff.

18 Für die Entwicklung der Wohlfahrtspflege im NS-Staat vgl. ebenda, S. 51ff., 83ff.; Hansen, Wohlfahrtspolitik. Zu den diversen Modifizierungen des Fürsorgerechts siehe Oestreicher, Entwicklung, S. $65 \mathrm{f}$. 
„Volkspflege“ und damit verbunden einen Umbau innerhalb des fürsorgerischen Apparates. So gewann die Gesundheitsfürsorge erheblich an Gewicht. Auf der anderen Seite bildete sich als gesondertes Tätigkeitsgebiet die „Asozialen-Fürsorge“ heraus, die zunächst auf Erfassung und Aussonderung der „Minderwertigen“, schließlich deren „Vernichtung“ zielte und immer mehr in die Hände der Polizei und SS überging.

Die Fürsorge für die „produktiven“ und „wertvollen Volksgenossen“ übernahm zunehmend die neu gegründete „Nationalsozialistische Volkswohlfahrt“ (NSV), die als zentralistischer Großverband der Partei immer mehr Kompetenzen an sich ziehen konnte, so daß der kommunalen Fürsorge in erster Linie die Aufgabe wirtschaftlicher Mindestsicherung der Empfänger traditioneller Armen- und gehobener Fürsorge verblieb, die infolge sinkender offener Arbeitslosigkeit und immer selektiverer Fürsorgegewährung ohnehin an Bedeutung verlor. ${ }^{19}$

Entsprechend der allgemeinen Entmachtung der Gemeinden während der NSZeit manifestierte sich der zunehmende Bedeutungsverlust der kommunalen Wohlfahrtspflege bereits 1934, als die Gesundheitsfürsorge als zentrales Betätigungsfeld der NS-Rassenideologie weitgehend verstaatlicht wurde. ${ }^{20}$ Diesem bald erheblich ausgebauten öffentlichen Gesundheitsdienst oblag neben der Pflege und gesundheitshygienischen Erziehung der „Erbgesunden“ auch als pervertiertes Komplement die Kontrolle und Hilfe bei der Durchführung der „Maßnahmen zur Verhütung erbkranken Nachwuchses“.

Naturgemäß betraf die Expansion der NSV, die sich selbst als freier Wohlfahrtsverband definierte, schon ganz früh und unmittelbar die bisherigen Spitzenverbände der freien Wohlfahrtspflege, gegenüber denen die NSV die Führungsrolle beanspruchte: Während die Arbeiterwohlfahrt (AWO) bereits 1933 verboten wurde und ihr Vermögen später an die NSV überging, konnten die beiden christlichen Verbände unter erschwerten Bedingungen weiterarbeiten; wenn sie später auch erhebliche Einbußen bei der Jugendhilfe (Kindergärten etc.) hinnehmen mußten, konnten sie zumindest ihre klassische Domäne der Anstaltsfürsorge behaupten. ${ }^{21}$

19 Vgl. Hansen, Wohlfahrtspolitik, S.118ff., 325ff.; Sachße/Tennstedt, Geschichte, Bd.3, S. $91 \mathrm{ff} . ;$ Hammerschmidt, Wohlfahrtsverbände im NS-Staat, S.366ff.

20 "Gesetz über die Vereinheitlichung des Gesundheitswesens“ (GVG) vom 3. Juli 1934, RGBl. 1934 I, S. 531; vgl. ausführlich Labisch/Tennstedt, Weg, S. 197ff.

$21 \mathrm{Vgl}$. dazu insgesamt Hammerschmidt, Wohlfahrtsverbände im NS-Staat, S.135ff. Der Zentralwohlfahrtsstelle der deutschen Juden wurde zunächst der Status als freier Spitzenverband aberkannt; 1939 wurde sie aufgelöst und als Abteilung „Fürsorge“ in die „Reichsvereinigung der Juden in Deutschland“ überführt und stellte schließlich 1943 ihre Arbeit ein. Der Deutsche Paritätische Wohlfahrtsverband (DPW) wurde Ende 1933 aufgelöst, seine Mitgliedsorganisationen wurden der NSV unterstellt. Nachdem 1937 die Rotkreuz-Einzelorganisationen zum Deutschen Roten Kreuz (DRK) zusammengefaßt worden waren, ging der Sanitätsdienst des DRK im Zweiten Weltkrieg an die Wehrmacht über, und das DRK wurde neben allgemeinen krankenpflegerischen Aufgaben zuständig für die Betreuung von Kriegsgefangenen sowie für Hilfsmaßnahmen für die Zivilbevölkerung in den besetzten Gebieten; vgl. ebenda, S. 157ff.; Buck, Entwicklung, S. 144, $162,165$. 
Während des Zweiten Weltkrieges wurde neben einigen Vereinfachungsverordnungen auf Druck der NSV und gegen den Widerstand der Kommunen 1941 eine Bestimmung erlassen, die grundsätzliche Bedeutung für die weitere Entwicklung der Fürsorge gewann: Der Runderlaß über „Öffentliche Fürsorge, insbesondere Aufbau der Richtsätze“ vom 31. Oktober $1941^{22}$ schrieb erstmalig rechtlich zwingend in ihren Komponenten vereinheitlichte, regional einander angeglichene und am tatsächlichen Bedarf orientierte Richtsätze vor, beschnitt dadurch den „vorherrschenden kommunalen Unterstützungswildwuchs“ 23 und bildete eine „entscheidende Stufe der Durchsetzung des [...] standardisierten nationalen Existenzminimums“24. Darüber hinaus erweiterte der Erlaß den Kreis der Bezieher gehobener Fürsorge auf die Gruppe der „Erbtüchtigen“ und machte damit die gehobene Fürsorge de facto zum „Regelfall“ einer nun ebenfalls den Prinzipien der „Rassenpflege“ verpflichteten öffentlichen Fürsorge. ${ }^{25}$

In der fürsorgerischen Praxis konnte die NSV während des Krieges auch durch die Übernahme neuer Aufgaben hinter der Front und bei der Katastrophenhilfe auf Kosten der Kommunen weiter expandieren. ${ }^{26}$ Immerhin gewannen die Kommunen, die jetzt auch für die kriegsbedingten Notaufgaben wie die Versorgung ihrer Einwohner mit Nahrung, Kleidung und Heizmaterial, Unterbringung und Evakuierung von Ausgebombten und ersten Flüchtlingen zuständig waren, zumindest gegenüber den staatlichen Instanzen einen Teil ihrer administrativen Selbständigkeit zurück ${ }^{27}$ und waren nach dem Zusammenbruch der Reichs- wie der Parteiinstitutionen in der Lage, die nun ihnen zunächst allein überlassenen fürsorgerischen Aufgaben im Rahmen des Möglichen tatsächlich zu übernehmen.

\footnotetext{
22 RMBliV. 1941, S. 1951.

23 Rudloff, Fürsorge, S. 195.

${ }^{24}$ Leibfried/Hansen/Heisig, Geteilte Erde, S. 169; vgl. Sachße/Tennstedt, Geschichte, Bd.3, S. $246 \mathrm{ff}$.

${ }^{25}$ Vgl. Sachße/Tennstedt, Geschichte, Bd. 3, S. 250; ferner Rudloff, Fürsorge, S. 195.

26 Vgl. Hansen, Wohlfahrtspolitik, S. 197ff., $325 \mathrm{ff}$.

27 Vgl. Mutius, Kommunalverwaltung, 1985, S. $1079 \mathrm{ff}$.
} 